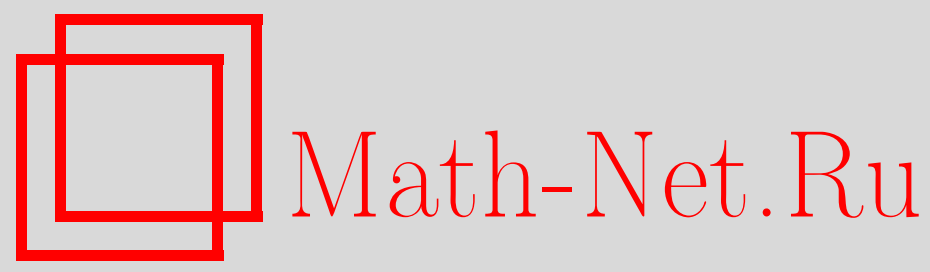

А. Б. Шидловский, О свойствах алгебраических уравнений, связывающих множество $E$-функций над полем рациональных функций, Матем. заметки, 2000, том 68, выпуск $5,761-770$

DOI: https://doi.org/10.4213/mzm996

Использование Общероссийского математического портала Math-Net.Ru подразумевает, что вы прочитали и согласны с пользовательским соглашением http://www . mathnet.ru/rus/agreement

Параметры загрузки:

IP : 35.173 .219 .12

26 апреля 2023 г., 13:57:44

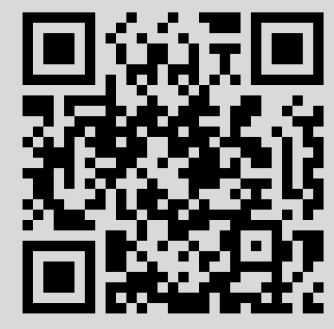




\section{О СВОЙСТВАХ АЛГЕБРАИЧЕСКИХ УРАВНЕНИЙ, СВЯЗЫВАЮЩИХ МНОЖЕСТВО Е-ФУНКЦИЙ НАД ПОЛЕМ РАЦИОНАЛЬНЫХ ФУНКЦИЙ}

\section{А. Б. Шидловский}

В работе доказываются теоремы о линейной и алгебраической независимости значений в алгебраических точках множества $E$-функций, связанных алгебраическими уравнениями над полем рациональных функций, и теорема об оценке модуля многочлена с цельми коэффициентами от значения таких функций. При этом используются свойства идеала в кольце многочленов от нескольких переменных, образованного уравнениями, связывающими рассматриваемые функции над полем рациональных функций.

Библиографиия: 7 названий.

Рассматривается множество $E$-функций, алгебраически зависимых над $\mathbb{C}(z)$. Доказываются некоторые утверждения об арифметических свойствах их значений в алгебраических точках. При этом используются свойства идеала в кольце $\mathbb{C}\left[z, z_{1}, \ldots, z_{m}\right]$, образованного всеми алгебраическими уравнениями, связывающими рассматриваемые функции над $\mathbb{C}(z)$.

Всюду в дальнейшем

$$
f_{1}(z), \ldots, f_{m}(z)
$$

будет совокупность $E$-функций, составляюших решение системы линейных однородных дифференциальных уравнений

$$
y_{k}^{\prime}=\sum_{i=1}^{m} Q_{k, i} y_{i}, \quad k=1, \ldots, m, \quad m \geqslant 2, \quad Q_{k, i} \in \mathbb{C}(z),
$$

или системы

$$
y_{k}^{\prime}=Q_{k, 0}+\sum_{i=1}^{m} Q_{k, i} y_{i}, \quad k=1, \ldots, m, \quad m \geqslant 1,
$$

a $T(z) \in \mathbb{C}[z]$ обозначает наименьший общий знаменатель всех функций $Q_{k, i}$ в системе (2) или (3).

Рассмотрим множество значений функций (1)

$$
f_{1}(\xi), \ldots, f_{m}(\xi)
$$

Работа выполнена при частичной финансовой поддержке Российского фонда фундаменталных исследований, грант № 97-01-00181, и объединенного проекта фонда INTAS и Российского фонда фундаментальных исследований, грант № IR-97-1904. 
в точке $\xi \in \mathbb{A}, \xi T(\xi) \neq 0$, где $\mathbb{A}$ - поле всех алгебраических чисел.

Доказано, что числа (4) однородно алгебраически независимы (алгебраически независимы) тогда и только тогда, когда функции (1) однородно алгебраически независимы (алгебраически независимы) над $\mathbb{C}(z)$. Если же функции (1) алгебраически зависимы над $\mathbb{C}(z)$, то в ряде случаев доказана алгебраическая независимость некоторых подсовокупностей чисел (4). Установлено, что степени однородной трансцендентности (степени трансцендентности) функций (1) над $\mathbb{C}(z)$ и чисел (4) над $\mathbb{A}$ равны.

С определением $E$-функций, историей вопроса и основными результатами об арифметических свойствах значений $E$-функций можно ознакомиться по книге [1].

В дальнейшем все рассуждения будут проводиться только в однородных случаях. Также некоторые утверждения формулируются только в однородных случаях. Соответствующие неоднородные аналоги следуют из однородных, если заменить $m$ на $m+1$ и ввести функцию $f_{m+1}(z)=1$.

Пусть $\xi \in \mathbb{C}$, а $\varphi_{\xi}: \mathbb{C}\left[z, z_{1}, \ldots, z_{m}\right] \rightarrow \mathbb{C}\left[z_{1}, \ldots, z_{m}\right]$ - гомоморфизм, которьй представляет собой замену переменной $z$ на $\xi$ во всех многочленах кольца $\mathbb{C}\left[z, z_{1}, \ldots, z_{m}\right]$. Если $\mathfrak{U}$ - любой идеал этого кольца, то будем обозначать через $\mathfrak{U}_{\xi}=\varphi_{\xi}(\mathfrak{U})$ идеал, соответствующий $\mathfrak{U}$ в кольце $\mathbb{C}\left[z_{1}, \ldots, z_{m}\right]$ при гомоморфизме $\varphi_{\xi}$.

Пусть $\mathfrak{P}$ - простой идеал кольца $\mathbb{C}\left[z, z_{1}, \ldots, z_{m}\right]$, порожденный всеми однородными по $z_{1}, \ldots, z_{m}$ многочленами, обращающимися в нуль при подстановке в них вместо $z_{1}, \ldots, z_{m}$ функций (1). (Если функции (1) однородно алгебраически независимы над полем рациональных функций, то полагаем $\mathfrak{P}=(0)$.)

Всюду в дальнейшем число $\xi \in \mathbb{A}, \xi T(\xi) \neq 0$, будет фиксированным, а $\mathbb{K}_{0}$ обозначает алгебраическое поле над $\mathbb{Q}$, содержащее $\xi$ и все коэффициенты степенньх рядов по степеням $z$ функций (1).

Если однородньй идеал (идеал) $\mathfrak{P}_{\xi}$, соответствующий функциям $(1)$, является простым, то совокупность функций (1) будем называть однородно простой (простой) в mочке $\xi$.

Пусть $\mathbb{L}_{N}$ - линейное пространство над $\mathbb{C}(z)$, порожденное произведениями степеней функций (1)

$$
f_{m}^{k_{m}}(z) \cdots f_{1}^{k_{1}}(z), \quad k_{1}+\cdots+k_{m}=N, \quad N \in \mathbb{N},
$$

a $\mathbb{L}_{N, \xi}$ - линейное пространство над $\mathbb{A}$, порожденное значениями функций (5) в точке $\xi$. Обозначим $r_{N}=\operatorname{dim} \mathbb{L}_{N}$ и $\rho_{N}=\operatorname{dim} \mathbb{L}_{N, \xi}$.

В этой статье будем назьвать $\xi$-базисом $\mathbb{L}_{N}$ множество функций

$$
F_{1}(z), \ldots, F_{r_{N}}(z)
$$

удовлетворяющих условиям:

1) функции (6) составляют подмножество множества функций (5);

2) функции (6) линейно независимы над $\mathbb{C}(z)$;

3) каждая из функций (5) линейно выражается через функции (6) с коэффициентами из $\mathbb{K}_{0}(z)$, не имеющими полюсов в точке $\xi$.

При любом $N \xi$-базисы существуют (см. [1, гл. 4 , леммы 1 и 2 ; гл. 3 , лемма 2]).

ТЕОрема 1. Пусть совокупность Е-функиий (1) составляет решение системы линейных однородных дифференциальных уравнений (2) и является однородно простой в точке $\xi \in \mathbb{A}, \xi T(\xi) \neq 0$, a $N \in \mathbb{N}$ и функиии (6) составляют $\xi$-базис линейного пространства $\mathbb{L}_{N}$, порохденного произведениями степеней (5) над $\mathbb{C}(z)$. 
Тогда числа

$$
F_{1}(\xi), \ldots, F_{r_{N}}(\xi)
$$

линейно независимь над $\mathbb{A}$.

Из теоремы 1 при ее условиях получаем очевидные следствия.

СлЕДСТВИЕ 1. Кажддй $\xi$-базис линейного пространства $\mathbb{L}_{N}$ при подстановке $z=\xi$ переходит в базис линейного пространства $\mathbb{L}_{N, \xi}$.

СЛЕДСТВИЕ 2. Имеет место равенство $r_{N}=\rho_{N}$.

В [1, с. 161-162] приводятся примеры некоторых случаев, когда выполняются утверждения, аналогичные следствиям 1 и 2, и указьвается на трудности доказательства или опровержения их в общем случае.

Пусть в дальнейшем $\mathbb{K}$ обозначает алгебраическое поле над $\mathbb{Q}$, содержащее поле $\mathbb{K}_{0}$ и все коэффициенты однородного многочлена $P$,

$$
P=P\left(z_{1}, \ldots, z_{m}\right) \in \mathbb{K}\left[z_{1}, \ldots, z_{m}\right], \quad \operatorname{deg}_{\bar{z}} P \geqslant 1
$$

a $h=[\mathbb{K}: \mathbb{Q}]$. Далее, пусть $\mathbb{K}_{i}, i=1, \ldots, h,-$ алгебраические поля, сопряженные с полем $\mathbb{K} ; \xi_{i}, i=1, \ldots, h,-$ числа, сопряженные с $\xi$;

$$
f_{1, i}(z), \ldots, f_{m, i}(z), \quad i=1, \ldots, h
$$

- функции, получающиеся из функций (1) заменой всех коэффициентов их степенных рядов по степеням $z$ на числа, сопряженные из поля $\mathbb{K}_{i} ;$ а $P_{i}=P_{i}\left(z_{1}, \ldots, z_{m}\right), i=1, \ldots, h,-$ многочлены, получающиеся аналогично из многочлена $P$.

Установим вспомогательные предложения для доказательства теоремы 1.

Лемма 1. Однородный многочлен $P$ из (8) принадлежсит идеалу $\mathfrak{P}_{\xi}$ тогда и только тогда, когда существуют однородные по $z_{1}, \ldots, z_{m}$ многочлены $Q=Q(z$, $\left.z_{1}, \ldots, z_{m}\right) u R=R\left(z, z_{1}, \ldots, z_{m}\right), Q, R \in \mathbb{K}\left[z, z_{1}, \ldots, z_{m}\right]$, такие, что

$$
P=(z-\xi) Q+R, \quad R \in \mathfrak{P} \text {. }
$$

ДокАЗАТЕЛЬСТво. Если имеет место равенство (10), то, полагая в нем $z=\xi$, получим, что

$$
P\left(z_{1}, \ldots, z_{m}\right)=R\left(\xi, z_{1}, \ldots, z_{m}\right)
$$

и, следовательно, $P \in \mathfrak{P}_{\xi}$.

Пусть теперь многочлен $P \in \mathfrak{P}_{\xi}$. По определению идеала $\mathfrak{P}_{\xi}$ вьполняется равенство $(10)$, где однородные по $z_{1}, \ldots, z_{m}$ многочлены $Q, R \in \mathbb{C}\left[z, z_{1}, \ldots, z_{m}\right]$. Докажем, что $Q$ и $R$ можно выбрать из кольца $\mathbb{K}\left[z, z_{1}, \ldots, z_{m}\right]$.

Пусть $\mathscr{L}$ обозначает линейное пространство над $\mathbb{K}$, порожденное коэффициентами многочлена $R$. Покажем, что $1 \in \mathscr{L}$.

Выберем числа $\alpha_{1}, \ldots, \alpha_{m} \in \mathbb{K}$ такими, что $P\left(\alpha_{1}, \ldots, \alpha_{m}\right) \neq 0$, и подставим в равенство (10) $z=\xi$ и $z_{k}=\alpha_{k}, k=1, \ldots, m$. Тогда $P\left(\alpha_{1}, \ldots, \alpha_{m}\right)=R\left(\xi, \alpha_{1}, \ldots, \alpha_{m}\right)$. Из последнего равенства следует, что число 1 есть линейная комбинация коэффищиентов многочлена $R$ с коэффициентами из $\mathbb{K}$. Следовательно, $1 \in \mathscr{L}$. 
Выберем в $\mathscr{L}$ базис $b_{1}, \ldots, b_{N}$ такой, что $b_{1}=1$. Тогда

$$
R=\sum_{l=1}^{N} b_{l} R_{l}, \quad R_{l} \in \mathbb{K}\left[z, z_{1}, \ldots, z_{m}\right], \quad l=1, \ldots, N .
$$

Taк как $R \in \mathfrak{P}$, то

$$
R\left(z, f_{1}(z), \ldots, f_{m}(z)\right)=0
$$

Докажем, что

$$
R_{l} \in \mathfrak{P}, \quad l=1, \ldots, N .
$$

Из равенств (12) и (13) следует, что

$$
R\left(z, f_{1}(z), \ldots, f_{m}(z)\right)=\sum_{l=1}^{N} b_{l} R_{l}\left(z, f_{1}(z), \ldots, f_{m}(z)\right)=0 .
$$

Поэтому

$$
\left.\sum_{l=1}^{N} b_{l}\left(\frac{d}{d z}\right)^{k} R_{l}\left(z, f_{1}(z), \ldots, f_{m}(z)\right)\right|_{z=0}=0, \quad k=0,1,2, \ldots
$$

Поскольку

$$
\left.\left(\frac{d}{d z}\right)^{k} R_{l}\left(z, f_{1}(z), \ldots, f_{m}(z)\right)\right|_{z=0} \in \mathbb{K}, \quad k=0,1,2, \ldots,
$$

то ввиду линейной независимости над $\mathbb{K}$ чисел $b_{1}, \ldots, b_{N}$ из равенства $(15)$ получаем, что

$$
\left.\left(\frac{d}{d z}\right)^{k} R_{l}\left(z, f_{1}(z), \ldots, f_{m}(z)\right)\right|_{z=0}=0, \quad k=0,1,2, \ldots,
$$

и

$$
R_{l}\left(z, f_{1}(z), \ldots, f_{m}(z)\right)=0, \quad l=1, \ldots, N .
$$

Следовательно, вьполняются условия (14).

После деления многочлена $R_{l}$ на $z-\xi$ с остатком имеем равенства

$$
R_{l}=(z-\xi) T_{l}+S_{l}, \quad l=1, \ldots, N,
$$

где $T_{l} \in \mathbb{K}\left[z, z_{1}, \ldots, z_{m}\right]$, а $S_{l} \in \mathbb{K}\left[z_{1}, \ldots, z_{m}\right]$. Из равенств $(12)$ и (16) находим, что

$$
R=\sum_{l=1}^{N} b_{l}\left((z-\xi) T_{l}+S_{l}\right)
$$

Подставляя это значение $R$ в равенство (10), приходим к равенству

$$
P=(z-\xi) Q+\sum_{l=1}^{N} b_{l}\left((z-\xi) T_{l}+S_{l}\right),
$$

из которого при $z=\xi$ имеем, что

$$
P=\sum_{l=1}^{N} b_{l} S_{l}
$$

Сравнивая в равенстве (17) коэффициенты при различных произведениях степеней переменных $z_{1}, \ldots, z_{m}$, находим, что $P=S_{1}, S_{2}=\cdots=S_{N}=0$.

Полагая в первом из равенств (16) $S_{1}=P, T_{1}=-Q, R_{1}=R$, получаем равенство (10), в котором $Q$ и $R$ удовлетворяют утверждению леммы. 
Лемма 2. Однородный многочлен $P$ из (8) принадлежит $\mathfrak{P}_{\xi}$ в том и только том случае, когда выполняются равенства

$$
P_{i}\left(f_{1, i}\left(\xi_{i}\right), \ldots, f_{m, i}\left(\xi_{i}\right)\right)=0, \quad i=1, \ldots, h .
$$

ДоказАТЕльство. Если $P \in \mathfrak{P}_{\xi}$, то по лемме 1 вьполняется равенство (10), где однородные многочлены $Q, R \in \mathbb{K}\left[z, z_{1}, \ldots, z_{m}\right]$ и $R\left(\xi, z_{1}, \ldots, z_{m}\right) \neq 0$. Из равенства (10) имеем, что

$$
P_{i}=\left(z-\xi_{i}\right) Q_{i}+R_{i}, \quad i=1, \ldots, h,
$$

где многочлены $Q_{i}$ и $R_{i}$ получаются из многочленов $Q$ и $R$ заменой их коэффициентов на сопряженные числа из поля $\mathbb{K}_{i}$.

Так как $R \in \mathfrak{P}$, имеет место равенство (13). Поэтому

$$
R_{i}\left(z, f_{1, i}(z), \ldots, f_{m, i}(z)\right)=0, \quad i=1, \ldots, h,
$$

и

$$
R_{i}\left(\xi_{i}, f_{1, i}\left(\xi_{i}\right), \ldots, f_{m, i}\left(\xi_{i}\right)\right)=0, \quad i=1, \ldots, h .
$$

Но тогда при $z=\xi_{i}$ и $z_{k}=f_{k, i}\left(\xi_{i}\right), k=1, \ldots, m$, из равенств (19) следуют равенства (18).

Пусть теперь вьполняются равенства (18). Докажем, что $P \in \mathfrak{P}_{\xi}$.

Рассмотрим какой-либо $\xi$-базис (6) линейного пространства $\mathbb{L}_{N}$, где $N=\operatorname{deg}_{\bar{z}} P$. Из условия 3) его определения следует, что

$$
P\left(f_{1}(z), \ldots, f_{m}(z)\right)=S_{1} F_{1}(z)+\cdots+S_{r_{N}} F_{r_{N}}(z)
$$

где $S_{1}, \ldots, S_{r_{N}} \in \mathbb{K}(z)$ и не имеют полюсов в точке $z=\xi$. Если $H(z)$ - общий наименьший знаменатель функций $S_{1}, \ldots, S_{r_{N}}$, то из равенства $(20)$ имеем, что

$$
H(z) P\left(f_{1}(z), \ldots, f_{m}(z)\right)=M_{1} F_{1}(z)+\cdots+M_{r_{N}} F_{r_{N}}(z),
$$

где $H(z), M_{1}, \ldots, M_{r_{N}} \in \mathbb{K}[z]$ и $H(\xi) \neq 0$.

Из условий 1)-3) определения $\xi$-базиса и леммы 18 из [1, гл. 3] получаем, что совокупность $r_{N} E$-функций (6) составляет решение системы из $r_{N}$ линейных однородных дифференциальных уравнений с коэффициентами из $\mathbb{K}(z)$, не имеющими полюсов в точке $z=\xi$, и линейно независима над $\mathbb{K}(z)$. Поэтому, предполагая, что не все многочлены $M_{1}, \ldots, M_{r_{N}}$ обращаются в нуль в точке $z=\xi$ и применяя к линейной форме, стоящей в правой части равенства $(21)$, теорему 1 из $[1$, гл. $11, \S 2]$, убеждаемся в том, что все равенства (18) вьполняться не могут. Поэтому при $z=\xi M_{j}=0, j=1, \ldots, r_{N}$, a равенство (21) имеет вид

$$
H(z) P\left(f_{1}(z), \ldots, f_{m}(z)\right)=(z-\xi) Q\left(z, f_{1}(z), \ldots, f_{m}(z)\right),
$$

где $Q\left(z, z_{1}, \ldots, z_{m}\right) \in \mathbb{K}\left[z, z_{1}, \ldots, z_{m}\right]$. Обозначим

$$
H(\xi) R\left(z, z_{1}, \ldots, z_{m}\right)=H(z) P\left(z_{1}, \ldots, z_{m}\right)-(z-\xi) Q\left(z, z_{1}, \ldots, z_{m}\right) .
$$

Из равенств (22) и (23) получаем равенство (13), а при $z=\xi$ из равенства (23) равенство (11), из которого следует, что $P \in \mathfrak{P}_{\xi}$. Лемма доказана. 
Лемма 3 (см. [2, теорема 1]). Если совокупность Е-функиий (1) является однородно простой в точке $\xi, \xi \in \mathbb{A}, \xi T(\xi) \neq 0$, то для любого однородного многочлена $Р$ из (8) такого, что $P \notin \mathfrak{P}_{\xi}$, выполняется условие $P\left(f_{1}(\xi), \ldots, f_{m}(\xi)\right) \neq 0$.

ДоКАЗАТЕЛЬСтво тЕоремы 1. Допустим противное, что при некотором $N$

$$
\alpha_{1} F_{1}(\xi)+\cdots+\alpha_{r_{N}} F_{r_{N}}(\xi)=0
$$

где $\alpha_{1}, \ldots, \alpha_{r_{N}} \in \mathbb{A}$ и не все эти числа равны нулю. Пусть теперь поле $\mathbb{K}$ таково, что оно содержит все числа $\alpha_{1}, \ldots, \alpha_{r_{N}}$. Обозначим

$$
P\left(f_{1}(z), \ldots, f_{m}(z)\right)=\alpha_{1} F_{1}(z)+\cdots+\alpha_{r_{N}} F_{r_{N}}(z) .
$$

Тогда $P\left(z_{1}, \ldots, z_{m}\right)$ есть однородньй многочлен $(8)$ и

$$
P\left(f_{1}(\xi), \ldots, f_{m}(\xi)\right)=0 .
$$

Из равенства (24) по лемме 3 следует, что $P \in \mathfrak{P}_{\xi}$. Тогда по лемме 2 вьполняются равенства (18).

Как и при доказательстве леммы 2 , убеждаемся в том, что совокупность $E$-функций (6) удовлетворяет всем условиям теоремы 1 из [1, гл. 11, 2$]$. По этой теореме равенства (18) оказываются противоречивыми и, следовательно, равенство (24) невозможно. Полученное противоречие доказьвает, что числа (7) линейно независимы над $\mathbb{C}(z)$. Теорема доказана.

Пусть теперь степень однородной трансцендентности (трансцендентности) функций (1) над $\mathbb{C}(z)$ равна $l, 1 \leqslant l<m$, а функции

$$
f_{1}(z), \ldots, f_{l}(z)
$$

однородно алгебраически независимы (алгебраически независимы) над $\mathbb{C}(z)$. Доказано, что числа

$$
f_{1}(\xi), \ldots, f_{l}(\xi)
$$

однородно алгебраически независимы (алгебраически независимы) для всех $\xi \in \mathbb{A}$, за исключением конечного числа из них. В ряде случаев установлены теоремы об алгебраической независимости чисел (26) для некоторых подклассов $E$-функций в точно оговоренных точках $\xi$ (см. [1]). Представляет интерес дальнейшее исследование арифметических свойств чисел $(26)$.

Условимся в многочленах из $\mathbb{C}[z]\left[z_{1}, \ldots, z_{m}\right]$ и $\mathbb{C}\left[z_{1}, \ldots, z_{m}\right]$ считать члены упорядоченньми в порядке лексикографического расположения по степеням $z_{m}, \ldots, z_{1}$.

Рассмотрим совокупность однородных минимальных уравнений (минимальных уравнений) функций (1) над $\mathbb{C}(z)$ (см. [3] или $[1$, гл. $4, \S 10])$, и пусть $\Lambda$ - множество нулей их старших коэффициентов, а $\Phi_{N, j}(z), j=1, \ldots, r_{N},-$ элементы множеств $B_{N}$, определенных минимальными уравнениями функций (1).

ТЕОРема 2. Пусть совокупность Е-функиий (1) составляет решение системь линейных однородных дифференииальных уравнений (2) и является однородно простой в точке $\xi \in \mathbb{A}, \xi \notin \Lambda, \xi T(\xi) \neq 0$.

Тогда при любом $N \in \mathbb{N}$ числа

$$
\Phi_{N, j}(\xi), \quad j=1, \ldots, r_{N},
$$

линейно независимы над $\mathbb{A}$ и составляют базис линейного пространства $\mathbb{L}_{N, \xi}$. 
ДокАЗАТЕЛЬСтво. По лемме 17 из $[1$, гл. $4, \S 10]$ при любом $N$ множество $B_{N}$ составляет базис линейного пространства $\mathbb{L}_{N}$, причем каждое из произведений степеней (5) линейно выражается через элементы $B_{N}$ с коэффициентами из $\mathbb{K}_{0}(z)$, не имеющими полюсов в точке $\xi$. Следовательно, при любом $N$ множество $B_{N}$ образует $\xi$-базис линейного пространства $\mathbb{L}_{N}$. Поэтому по теореме 1 при любом $N$ числа (27) линейно независимы над $\mathbb{A}$ и составляют базис $\mathbb{L}_{N, \xi}$.

ТЕОРема 3. Пусть совокупность Е-функиий (1) составляет решение системь линейных однородных дифференииальных уравнений (2) (дифференциальных уравнений (3)) и имеет степень однородной трансцендентности (степень трансцендентности) над $\mathbb{C}(z)$ равную $l, 1 \leqslant l<m$, а функиии (25) однородно алгебраически независимы (алгебраически независимы) над $\mathbb{C}(z)$. Далее, $\xi \in \mathbb{A}, \xi \notin \Lambda, \xi T(\xi) \neq 0$, а совокупность функиий (1) является однородно простой (простой) в точке $\xi$.

Тогда числа (26) однородно алгебраически независимы (алгебраически независи$\mathcal{M b l})$.

ДокАЗАТЕЛЬСтво. Рассмотрим однородные минимальные уравнения функций (1) над $\mathbb{C}(z)$ и определенные ими множества $B_{N}$. Поскольку при любом $N \in \mathbb{N}$ множество $B_{N}$ содержит элементы

$$
f_{l}^{k_{l}}(z) \cdots f_{1}^{k_{1}}(z), \quad k_{1}+\cdots+k_{l}=N
$$

то по теореме 2 числа (26) однородно алгебраически независимы.

Частньй случай теоремы 3, когда поле $\mathbb{K}_{0}$ есть мнимое квадратичное поле над $\mathbb{Q}$, в 1973 году установил В. Г. Чирский (см. [4]). В 1989 году автор статьи (см. [3]) доказал, что существует индекс $i, 1 \leqslant i \leqslant h$, такой, что числа $f_{1, i}\left(\xi_{i}\right), \ldots, f_{l, i}\left(\xi_{i}\right)$ однородно алгебраически независимы (алгебраически независимы).

В статье автора [5] рассматривается теорема 3 , аналогичная теореме 3 настоящей работы. Но там доказательство проводится недостаточно подробно. Утверждения леммы 2 и установленной ниже леммы 5 дополняют доказательства этой теоремы. В той же статье доказательства следствий 3 и 4 ошибочны. Их утверждения можно рассматривать как гипотезы.

ТЕОрема 4. Пусть множество Е-функиий (1) составляет решение системь линейных однородных дифференииальных уравнений (2) (дифференциальных уравнений (3)) и имеет степень однородной трансцендентности (степень трансцендентности) над $\mathbb{C}(z)$ равную $l, 1 \leqslant l \leqslant m ; \xi \in \mathbb{A}, \xi T(\xi) \neq 0 ; \mathbb{K}-$ какое-либо алгебраическое поле над $\mathbb{Q}$, содержащее поле $\mathbb{K}_{0}$, а совокупность Е-функиий (1) является однородно простой в точке $\xi$. Далее, однородный многочлен (многочлен)

$$
P=P\left(z_{1}, \ldots, z_{m}\right) \in \mathbb{Z}_{\mathbb{K}}\left[z_{1}, \ldots, z_{m}\right], \quad \operatorname{deg}_{\bar{z}} P=s, \quad s \geqslant 1, \quad|P|=H,
$$

где

Тогда либо

$$
P\left(f_{1}(\xi), \ldots, f_{m}(\xi)\right)=0
$$

либо

$$
\left|P\left(f_{1}(\xi), \ldots, f_{m}(\xi)\right)\right|>c_{0} H^{-\gamma_{0} h^{l} s^{l-1}}
$$


а в неоднородном случае

$$
\left|P\left(f_{1}(\xi), \ldots, f_{m}(\xi)\right)\right|>c H^{-\gamma h^{l+1} s^{l}},
$$

әде с $c_{0}>0$ и с>0 - постоянные, зависящие от функиий (1) и чисел $m, l, \xi$, а $\gamma_{0}>0$ и $\gamma>0$ - постоянные, зависящие только от функиий (1) и числа l.

Установим вспомогательные предложения для доказательства теоремы 4.

ЛЕмма 4. Идеал $\mathfrak{P}$ имеет базис, состоящий из многочленов кольиа $\mathbb{A}\left[z, z_{1}, \ldots\right.$, $\left.z_{m}\right]$.

ДокАЗАТЕЛЬСТво. Пусть $R_{1}, \ldots, R_{M} \in \mathbb{C}\left[z, z_{1}, \ldots, z_{m}\right]$ - какой-либо базис идеала $\mathfrak{P}$. Тогда

$$
R_{j}\left(z, f_{1}(z), \ldots, f_{m}(z)\right)=0, \quad j=1, \ldots, M .
$$

Рассмотрим линейное пространство над $\mathbb{A}$, порожденное всеми коэффициентами многочленов $R_{1}, \ldots, R_{M}$, и обозначим его $\mathscr{L}$. Пусть $b_{1}, \ldots, b_{N}-$ базис $\mathscr{L}$. Тогда каждьй многочлен $R_{j}$ единственным способом представляется в виде

$$
R_{j}=\sum_{l=1}^{N} b_{l} Q_{j, l}, \quad Q_{j, l} \in \mathbb{A}\left[z, z_{1}, \ldots, z_{m}\right] .
$$

Докажем, что все многочлены $Q_{j, l} \in \mathfrak{P}$.

Из равенств $(30)$ и $(31)$ имеем, что

$$
R_{j}\left(z, f_{1}(z), \ldots, f_{m}(z)\right)=\sum_{l=1}^{N} b_{l} Q_{j, l}\left(z, f_{1}(z), \ldots, f_{m}(z)\right)=0, \quad j=1, \ldots, M .
$$

Дифференцируя $k$ раз $(k \geqslant 0)$ равенство $(32)$, получаем

$$
\begin{gathered}
\left.\left(\frac{d}{d z}\right)^{k} R_{j}\left(z, f_{1}(z), \ldots, f_{m}(z)\right)\right|_{z=0}=\left.\sum_{l=1}^{N} b_{l}\left(\frac{d}{d z}\right)^{k} Q_{j, l}\left(z, f_{1}(z), \ldots, f_{m}(z)\right)\right|_{z=0}=0 \\
k=0,1,2, \ldots, \quad j=1, \ldots, M
\end{gathered}
$$

Так как все коэффициенты степенных рядов функций (1) являются алгебраическими числами, то

$$
\begin{gathered}
\left.\left(\frac{d}{d z}\right)^{k} Q_{j, l}\left(z, f_{1}(z), \ldots, f_{m}(z)\right)\right|_{z=0} \in \mathbb{A}, \\
k=0,1,2, \ldots, \quad j=1, \ldots, M, \quad l=1, \ldots, N .
\end{gathered}
$$

Поэтому из равенств (33) следует, что

$$
\begin{gathered}
\left.\left(\frac{d}{d z}\right)^{k} Q_{j, l}\left(z, f_{1}(z), \ldots, f_{m}(z)\right)\right|_{z=0}=0, \\
k=0,1,2, \ldots, \quad j=1, \ldots, M, \quad l=1, \ldots, N .
\end{gathered}
$$

Следовательно, все $Q_{j, l}\left(z, f_{1}(z), \ldots, f_{m}(z)\right)=0$. Из последних равенств получаем, что

$$
Q_{j, l} \in \mathfrak{P}, \quad j=1, \ldots, M, l=1, \ldots, N .
$$

Равенства (31) и (34) позволяют утверждать, что $\mathfrak{P}=\left(Q_{1,1}, \ldots, Q_{M, N}\right)$. Это доказывает утверждение леммы. 
СлЕДСТВИЕ 1. Идеал $\mathfrak{P}_{\xi} \subset \mathbb{C}\left[z_{1}, \ldots, z_{m}\right]$ имеет базис, состоящий из многочленов кольиа $\mathbb{A}\left[z_{1}, \ldots, z_{m}\right]$.

Так как $\xi \in \mathbb{A}$, то утверждение очевидно.

СЛЕДСТВИЕ 2. Пусть

$$
\mathfrak{Q}=\mathfrak{P}_{\xi} \cap \mathbb{A}\left[z_{1}, \ldots, z_{m}\right]
$$

- идеал кольиа $\mathbb{A}\left[z_{1}, \ldots, z_{m}\right]$. Тогда идеал $\mathfrak{P}_{\xi}$ является расширением идеала (35) в кольио $\mathbb{C}\left[z_{1}, \ldots, z_{m}\right]$.

ДокАЗАТЕЛЬСТво очевидно, так как по следствию 1 идеалы (35) и $\mathfrak{P}_{\xi}$ имеют один и тот же базис.

Пусть $\mathfrak{P}^{(i)}, i=1, \ldots, h$, обозначают идеалы кольца $\mathbb{C}\left[z, z_{1}, \ldots, z_{m}\right]$, порожденные всеми однородньми по $z_{1}, \ldots, z_{m}$ многочленами, обращающимися в нуль при подстановке в них вместо $z_{1}, \ldots, z_{m}$ соответствующих функций $(9)$ при $i=1, \ldots, h$, a

$$
\mathfrak{P}_{\xi_{i}}^{(i)}, \quad i=1, \ldots, h
$$

- идеалы кольца $\mathbb{C}\left[z_{1}, \ldots, z_{m}\right]$, соответствующие идеалам $\mathfrak{P}^{(i)}$ при гомоморфизмах $\varphi_{\xi_{i}}$.

ЛЕмма 5. Если идеал $\mathfrak{P}_{\xi}$ прост, то просты и все идеаль (36).

ДоКАЗАТЕЛЬСтво. Рассмотрим идеал (35) и идеалы

$$
\mathfrak{Q}_{i}=\mathfrak{P}_{\xi_{i}}^{(i)} \cap \mathbb{A}\left[z_{1}, \ldots, z_{m}\right], \quad i=1, \ldots, h .
$$

Следствие 1 позволяет утверждать, что при любом $i=1, \ldots, h$ идеал $\mathfrak{P}_{\xi_{i}}^{(i)}$ имеет базис, состоящий из многочленов кольца $\mathbb{A}\left[z_{1}, \ldots, z_{m}\right]$. Но тогда $\mathfrak{P}_{\xi_{i}}^{(i)}$ является расширением идеала $\mathfrak{Q}_{i}$ в кольцо $\mathbb{C}\left[z_{1}, \ldots, z_{m}\right]$. Поскольку идеал $\mathfrak{P}_{\xi}$ прост, то идеал (35) также является простым. При любом $i=1, \ldots, h$ идеал (37) сопряжен с идеалом (35) и поэтому является простым.

Поле $\mathbb{A}$ алгебраически замкнуто и имеет характеристику 0. По теореме 39 из [6, гл. 7, $\S 12]$ любой идеал (37) абсолютно прост. Поэтому его расширениев кольцо $\mathbb{C}\left[z_{1}, \ldots, z_{m}\right]$, т.е. идеал $\mathfrak{P}_{\xi_{i}}^{(i)}$, также является простым идеалом. Лемма доказана.

ДокаЗАТЕЛЬСТво тЕОРЕМЫ 4 . Если $P \in \mathfrak{P}_{\xi}$, то по лемме 2 вьполняется равенство (28). Если же $P \notin \mathfrak{P}_{\xi}$, то по лемме $2 P_{i} \notin \mathfrak{P}_{\xi_{i}}^{(i)}, i=1, \ldots, h$. Поскольку по лемме 5 все множества функций $(9)$ при $i=1, \ldots, h$ однородно просты в соответствующих точках $\xi_{i}$, то по лемме $3 P_{i}\left(f_{1, i}\left(\xi_{i}\right), \ldots, f_{m, i}\left(\xi_{i}\right)\right) \neq 0, i=1, \ldots, h$. Но тогда по вспомогательной теореме из книги $[1$, гл. $12, \S 6]$ справедливо неравенство (29). Теорема доказана.

В работе [2] доказано, что если совокупность $E$-фукнций (1) является однородно простой в точке $\xi$ и эти функции линейно независимы над $\mathbb{C}(z)$, то числа (4) линейно независимы над $\mathbb{A}$. Из этого утверждения и леммы 5 получаем следующую теорему. 
ТЕОРема 5. Пусть совокупность Е-функиий (1) составляет решение системы линейных однородных дифференциальных уравнений (2), линейно независима над $\mathbb{C}(z)$ и является однородно простой в точке $\xi \in \mathbb{A}, \xi T(\xi) \neq 0$.

Тогда числа $f_{1, i}\left(\xi_{i}\right), \ldots, f_{m, i}\left(\xi_{i}\right)$ при любом значении $i=1, \ldots, h$ линейно независимын над $\mathbb{A}$.

Лемма 5 позволяет распространить утверждение теоремы 3 на множество чисел $f_{1, i}\left(\xi_{i}\right), \ldots, f_{l, i}\left(\xi_{i}\right)$ при любом $i=1, \ldots, h$.

Заметим, что идеал $\mathfrak{P}_{\xi}$ является простым для всех чисел $\xi \in \mathbb{C}$, за исключением конечного числа из них (см. [2]).

Оценка многочлена от значений $E$-функций (1), установленная в теореме 4 , была также доказана в 1995 году А.И. Галочкиным (см. [7]) другим методом при меньших предположениях относительно точки $\xi$.

\section{СПИСОК ЦИТИРОВАННОЙ ЛИТЕРАТУРЫ}

[1] Шидловский А. Б. Трансцендентные числа. М.: Наука, 1987.

[2] Нестеренко Ю. В., Шидловский А. Б. О линейной независимости значений $E$-функций // Матем. сб. 1996. Т. 187. № 8. С. 93-108.

[3] Шидловский А. Б. К проблеме алгебраической независимости значений $E$-функций в алгебраических точках // Матем. заметки. 1989. Т. 45. №1. С. 115-122.

[4] Чирский В. Г. Об арифметических свойствах значений аналитических функций, связанных алгебраическими уравнениями над полем рациональных функций // Матем. заметки. 1973. Т. 14. № 1. С. 83-94.

[5] Шидловский А. Б. Об арифметических свойствах значений $E$-функций // Матем. заметки. 1999. Т. 66. № 3. С. 252-258.

[6] Зарисский О., Самюэль П. Коммутативная алгебра. Т. 2. М.: ИЛ, 1963.

[7] Галочкин А. И. Оценки снизу многочленов от значений алгебраически зависимых $E$-функций // Фундамент. и прикл. матем. 1995. Т. 1. № 1. С. 305-309.

Московский государственный университет им. М.В. Ломоносова

Поступило

22.01 .2000 